\title{
Coordinate Systems Integration for Craniofacial Database from Multimodal Devices
}

\author{
Deni Suwardhi ${ }^{1,2}$, Halim Setan ${ }^{2}$, Albert K. Chong ${ }^{3}$ \& Zainul Rajion ${ }^{4}$ \\ ${ }^{1}$ Department of Geodesy and Geomatic Engineering, Faculty of Civil \& Engineering, \\ Institute of Technology Bandung, Indonesia \\ ${ }^{2}$ Department of Geomatic Engineering, Faculty of Geoinformation Science \& \\ Engineering, UTM Skudai, Malaysia. \\ ${ }^{3}$ School of Surveying, University of Otago. Dunedin, New Zealand. \\ ${ }^{4}$ School of Dental Sciences, University Sains Malaysia.
}

\begin{abstract}
This study presents a data registration method for craniofacial spatial data of different modalities. The data consists of three dimensional (3D) vector and raster data models. The data is stored in object relational database. The data capture devices are Laser scanner, CT (Computed Tomography) scan and CR (Close Range) Photogrammetry. The objective of the registration is to transform the data from various coordinate systems into a single 3-D Cartesian coordinate system. The standard error of the registration obtained from multimodal imaging devices using 3D affine transformation is in the ranged of $1-2 \mathrm{~mm}$. This study is a step forward for storing the craniofacial spatial data in one reference system in database.
\end{abstract}

Keywords: Craniofacial; Database; Registration; CT-Scan; Laser-Scan; Close Range Photogrammetry.

\section{Introduction}

The word craniofacial is derived from the word cranium, referring to the skull, and facial, referring to the face. Craniofacial is the most complicated part of the human body and pertaining to the head and face. In the craniofacial, there are many important organs like eyes, ears, mouth and the most important organ is the brain. A function of craniofacial is protecting the important organs. Another function is for appearance.

Craniofacial vary greatly in shape and size. When these variations are extreme, they are regarded as deformities, and the judgment is based on intuitive concepts of normality. Objective measurements can be used to supplement aesthetic impressions. Determination of the normality or abnormality by measurements obtained from the patients requires a system of quantitative criteria [1]. The normal range of measurement is given by the mean and its standard deviation.

Received March 8, 2005, revised May 11, 2005, accepted for publication May 15, 2005. 
Craniofacial reconstructive surgery is performed on patient with birth defects, developmental abnormalities, trauma or injury, infection, tumors, or disease. It is generally performed to improve function, but may also be done to approximate a normal appearance. The goals of reconstructive surgery differ from those of cosmetic surgery. Cosmetic surgery is performed to reshape normal structures of the body to improve the patient's appearance and selfesteem.

One important question in planning of craniofacial reconstructive surgery is how the patient should look like after the operation. Appearance should be as close as possible to the aspect he/she would have without any defects. The step to achieve this is to build a database containing sets of "normal" craniofacial data which allows for a comparison of the current shape of a patient with a typical "normal" shape which taking such factors as age and sex of the patient into consideration [2].

The research and development of craniofacial database and information system in Malaysia is on-going and an experimental working of data registration is reported in this study. Close Range (CR) photogrammetry cameras, laser scanner and Computed Tomography (CT) scan data are added to the data acquisition system to obtain soft-tissue and hard tissue surfaces and landmarks. The aim of this study is to integrate the spatial data from all devices into one standard coordinate system so that they should be analyzed together in database management system environment.

\section{$2 \quad$ Method}

The concept of the coordinate system integration into single coordinate system is shown in Figure 1. The main processes are surface based registration, point based registration and osteometric scaling. After post-processing in each modality, the registration process is started. In the first stage, the skin surface from laser scanner is registered to the skin surface from CT scanner, with a surface to surface registration method. The registration itself is performed semi automatically using ICP (iterative closest points) based software. The ICP algorithm was introduced by Besl and McKay [3] and Zhang [4]. In the next step, the skin surface acquired from laser scanner is used to replace the CTscanned facial surface.

In the second stage, data coming from the first stage is registered to CR photogrammetry coordinate system using landmark-based method. Landmarkbased methods are mostly used to find rigid or affine transformations [5]. Osteometric scaling is performed in the last stage to transform the data into standard coordinate system. 


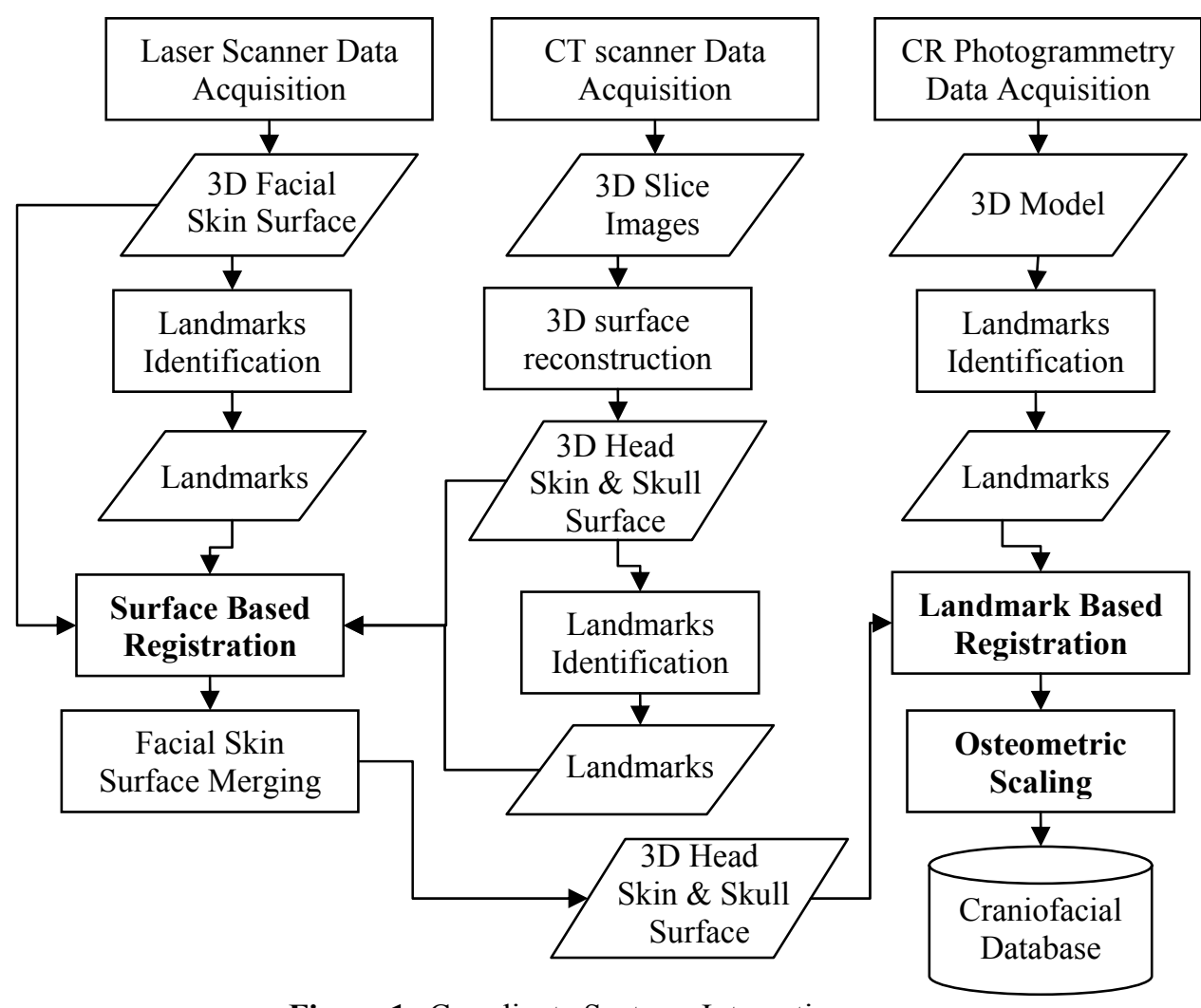

Figure 1 Coordinate Systems Integration.

\section{Data Acquisition}

The close range photogrammetry and laser triangulation techniques are applied to achieve good quality soft-tissue data. Six camera canon Powershoot S400 and two Minolta Vivid 910 laser scanners are used (see Fig. 2). In contrast to photogrammetry, laser scanning provides a very high point density on the object surface, within a more or less automatic recording procedure. This allows a very detailed surface description.

Additionally laser scanning is independent of texture information. The disadvantages of this measurement method lie in the measurement resolution and in the fact that laser scanner systems do not provide high quality RGBtexture information.

Two laser scanners are used to capture scanned data using Rapidform software. The patients sit on a chair with facial muscles relaxed whereas eyes opened and lips closed for seven seconds during the scanning. The distance of patient from 
the laser scanner was about 0.7 meter. At the same time, images of the human face are taken using normal digital camera (Fig. 2) to capture the texture.
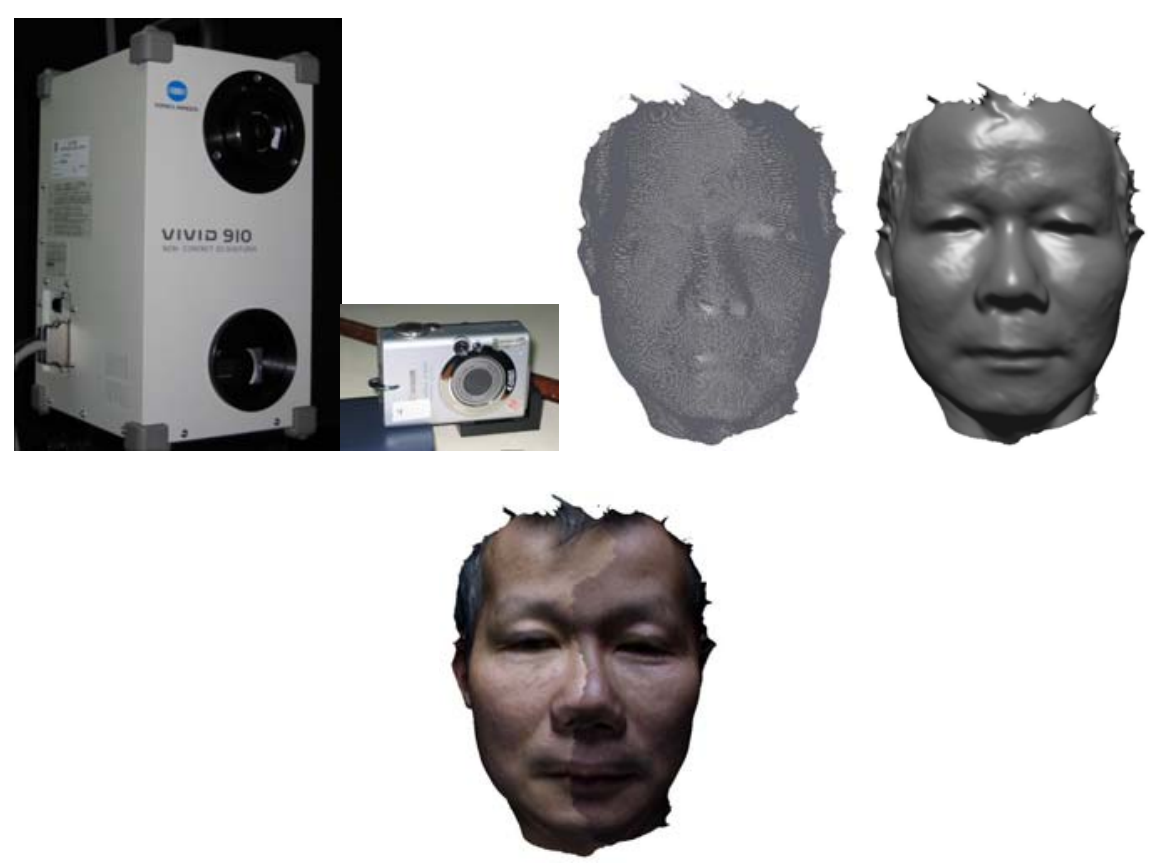

Figure 2 Vivid 910, Canon S400, and Laser Scanner.

Axial scans were obtained with a GE Lightspeed Plus CT Scanner System at the Department of Radiology, Hospital Universiti Sains Malaysia (HUSM) (Fig. 3).
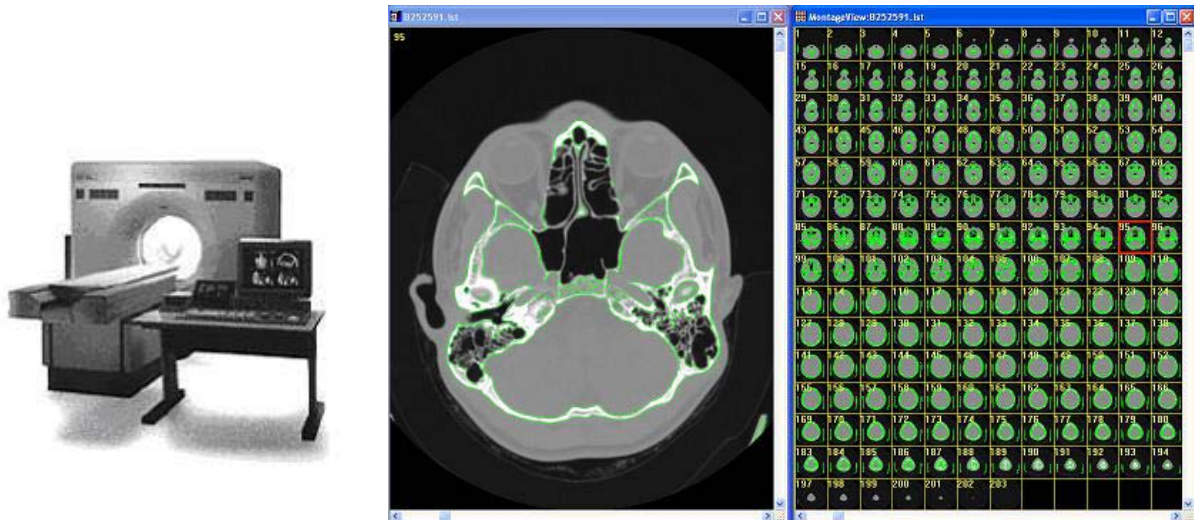

Figure 3 CT scanner and CT slice images. 


\section{D Surface Reconstruction}

After acquisition of CT images, image data is recorded in DICOM (Digital Imaging and Communications in Medicine) format. In DICOM files, detailed information on scanning parameters is recorded too. For example, slice thickness, pixel size on $\mathrm{x}$ and $\mathrm{y}$ directions, scanning type, scanning direction etc. In this study, 3D Slicer Software is used for 3D soft and hard-tissue reconstruction from $\mathrm{CT}$ images (Fig. 4).

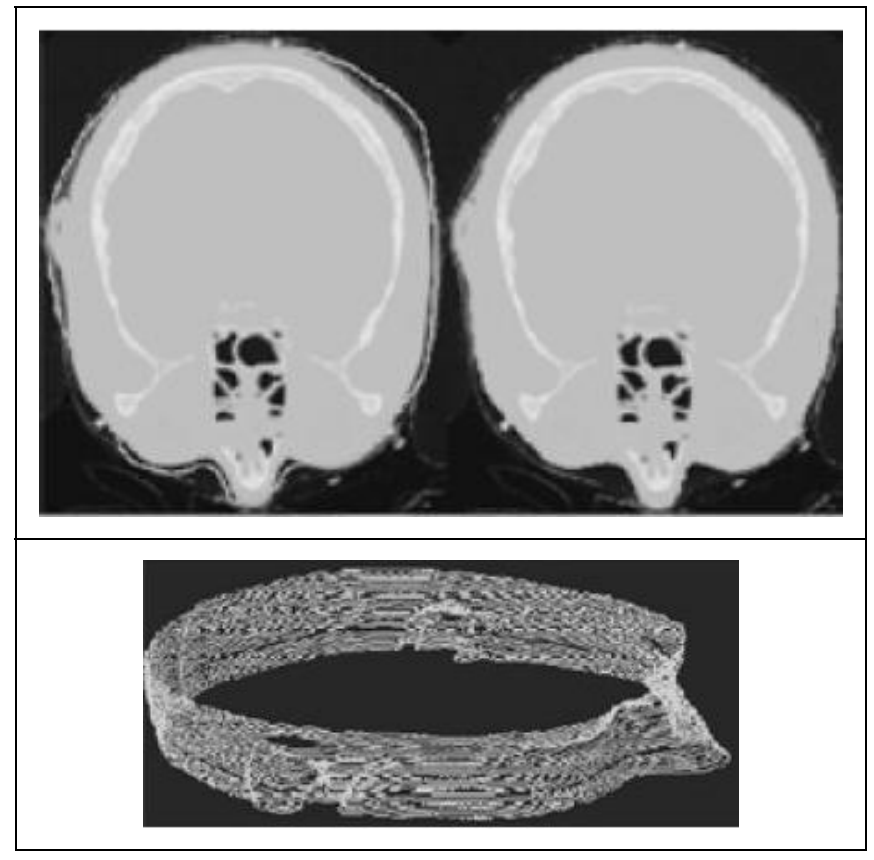

Figure 4 Image segmentation and its contours.

Before 3D surface reconstruction process, the data usually undergo some preprocessing for data conversion and possibly image filtering. From this point, one of several paths may be followed.

A 3D object is reconstructed from its contours on the cross-sectional images. The first step is to identify the different objects represented in the data volume so that they can be removed or selected for 3D reconstruction. The simplest way is to binarize the data with an intensity threshold, e.g., to distinguish bone from other tissues in CT [6] (Fig. 4).

After segmentation, rendering technique is used. An early approach for the reconstruction of the polygonal mesh from a stack of contours is based on the 
Delauney interpolation developed by Boissinnat [7]. A more recent method by Lorensen and Cline [8], called Marching Cubes algorithm, creates an isosurface, representing the locations of a certain intensity value in the data.

\section{Landmark identification}

The landmarks which are produced from this stage have two purposes: shape analysis and objects registration. Three basic landmarks are generally used:

1. Anatomical landmarks. Points assigned by an "expert" which correspond between organisms in some biologically meaningful way.

2. Mathematical landmarks. Points located on an object according to some mathematical or geometrical property of the object.

3. Pseudo landmarks. Points located either around the outline (2D) or between anatomical or mathematical landmarks.

For registration purpose, the anatomical and mathematical landmarks can be used as common points for objects to be registered. The positions of pseudo landmarks are not properly defined as sometime they are defined through interpolation. As a result, pseudo landmarks possess ambiguity if they are used as common points in registration process. Fig. 5 shows the anatomical landmarks on skin and skull [1][9].
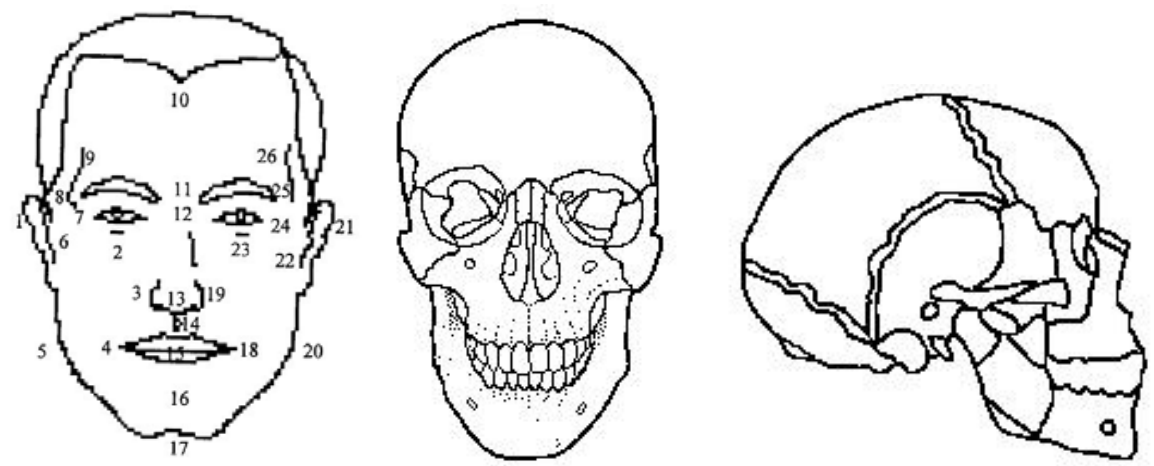

Figure 5 Landmarks location on skin and skull.

\section{Object Registration}

Registration is the determination of a one to one mapping or transformation between the coordinates in one space and those in another, such that points in the two spaces that correspond to the same anatomical point are mapped to each other. Registration of multimodal images makes it possible to combine different types of structural information (for example CT and MR) [10]. 
In this study, surface-based registration is accomplished by identifying known features common to two datasets. The application calculates an approximate position of one shell with respect to another using common geometric feature between two shells. When performing this operation, first selected shell is moved to second selected shell. Global registration exactly matches the position of all the selected shells at the same time using the overlapped region, which is automatically found. When the overlapped region is too little or the two registered shells are located far from each other, the registration result may be poor. Regional registration can align exactly two shells which having common region partially. The registration itself is performed semi automatically using ICP (iterative closest points) based software.

In the landmark-based registrations, both rigid body (3D similarity transformation) and non-rigid body (3D affine transformation) transformations are provided. If $3 \mathrm{D}$ objects which are to be registered, had been obtained by the same scanner with the same resolution, these objects could be matched with one transformation, two translations and one scale factor. If two object sets to be registered, are scanned with different resolution, then a non-rigid transformation is required. The transformation parameters are calculated with least squares adjustment. Anatomical or geometrical landmarks are used as being common points.

\section{Osteometric Scaling}

The 3-D Cartesian axis system as typically applied to head and face measurements is depicted in Fig. 6. The subject's head is oriented in a standard position called the Frankfort Horizontal Plane [1]. The head in this case is said to be in the Frankfort plane when the right and left porion and the infraobitales (OrL, OrR) (the lowest point on the anterior border of the bony eye socket) are aligned in a plane. The zero reference for the axes X, Y, and Z (PoC) lies at the center of the line connecting the right and left porion as shown in Fig 6. The $X$, $\mathrm{Y}$ and $\mathrm{Z}$ axes are pointed to the anterior, left and superior directions, respectively.

Shiang [11] performed osteometric scaling to normalize head spatial model of a set of similar specimens so that easy to analyze. Osteometric scaling describes a transformation from specimen landmarks to similar subject landmarks with a direct one-to-one relationship between the spatial coordinates of homologues. The technique is based on an affine coordinate transformation from landmark coordinates on a specimen body to homologue coordinates on a subject body. The transformation includes three-dimensional rigid body translation and rotation to provide alignment and orientation and also provides metric adjustments to allow for specimen-to-subject size differences. Scaling may be 
visualized as deformation, rotation and translation from an initial state (specimen) to a final state (subject).

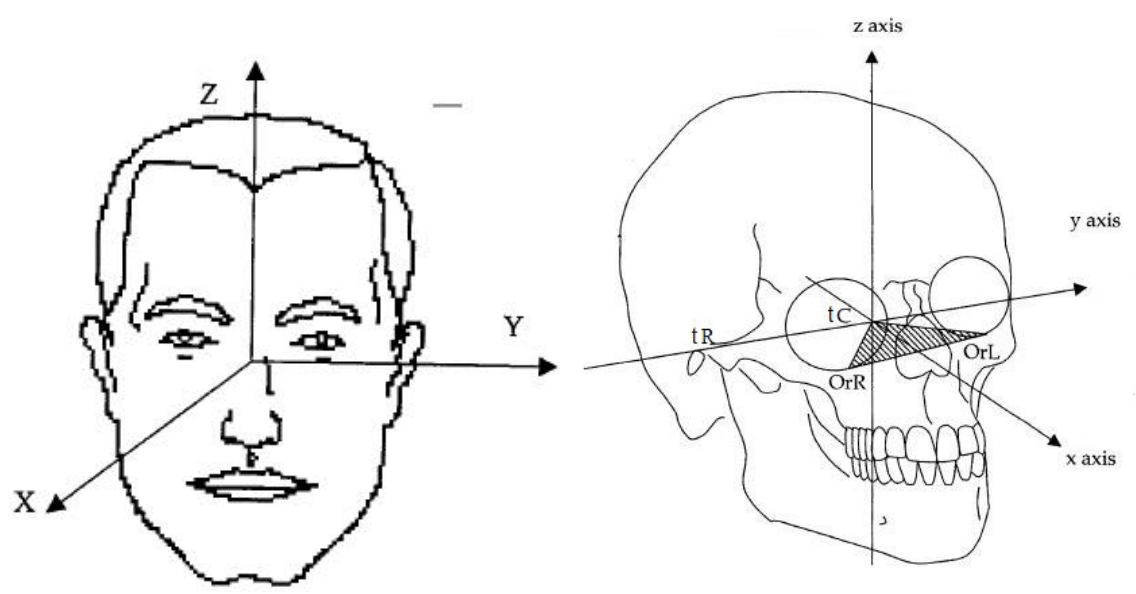

Figure 6 The coordinate system of Frankfort Horizontal Plane.

\section{$3 \quad$ Algorithm and Mathematical Model}

\section{Iterative Closest Point}

Besl and McKay [3] and Zhang [4] described a general-purpose, representation independent method for accurate and computationally efficient registration of one 3D shapes to another. The 3D shapes could include points, free-form curves and surfaces. This iterative approach (ICP) can be decomposed into two separate procedures:

1. For each point on shape $S_{1}$, locate the closest point on the other shape $S_{2}$.

2. Move $S_{1}$ by a rigid transformation which minimizes the mean square pairwise distance between each point on $S_{1}$ and its closest point on $S_{2}$.

Mathematically, the registration problem is to find the Euclidean transformation

$$
T: R^{n} \rightarrow R^{n}
$$

which minimizes the distance between two shapes $S_{1}$ and $S_{2}$

$$
\operatorname{dist}\left(S_{2}, T\left(S_{1}\right)\right)
$$


Given points $X_{1}=\left\{q_{1}, q_{2}, \ldots, q_{n}\right\}$ from shape $S_{1}$ and their corresponding closest points $X_{2}=\left\{p_{1}, p_{2}, \ldots, p_{n}\right\}$ from shape $S_{2}$, a Monte Carlo approximation of the distance between $S_{1}$ and $S_{2}$ is

$$
\sum_{i=1}^{n}\left\|p_{i}-q_{i}\right\|^{2}
$$

Thus, a Monte Carlo estimate of $T$ is a rigid transformation matrix that minimizes

$$
\sum_{i=1}^{n}\left\|p_{i}-T\left(q_{i}\right)\right\|^{2}
$$

Least Square method provides an explicit solution to the rigid transformation matrix T.

\section{3-D Affine and Similarity Transformation}

3-D Affine Transformation for landmark-based registration to Photogrammetry Coordinate System:

$$
\begin{aligned}
& X=x m_{11}+y \cdot m_{12}+z \cdot m_{13}+T_{x} \\
& Y=x \cdot m_{21}+y \cdot m_{22}+z \cdot m_{23}+T_{y} \\
& Z=x \cdot m_{31}+y \cdot m_{32}+z \cdot m_{33}+T_{z}
\end{aligned}
$$

where $x, y, z$ is $\mathrm{CT}+$ Laser Scan Coordinate System, and $X, Y, Z$ is Photogrammetry Coordiante System.

3-D Similarity Transformation for Osteometric Scaling to Frankfort Horizontal Coordinate System:

$$
\begin{aligned}
& X=\lambda \cdot\left(x \cdot r_{11}+y \cdot r_{12}+z \cdot r_{13}\right)+T_{x} \\
& Y=\lambda \cdot\left(x \cdot r_{21}+y \cdot r_{22}+z \cdot r_{23}\right)+T_{y} \\
& Z=\lambda \cdot\left(x \cdot r_{31}+y \cdot r_{32}+z \cdot r_{33}\right)+T_{z}
\end{aligned}
$$

where $x, y, z$ is $\mathrm{CT}+$ Laser Scan+Photogrammetry Coordinate System, and $X, Y, Z$ is Frankfort Horizontal Coordinate System.

In the matrix form, the equations (1) can be stated as:

$$
B=A X
$$




$$
B=\left[\begin{array}{c}
X_{1} \\
Y_{1} \\
Z_{1} \\
X_{2} \\
Y_{2} \\
\vdots \\
X_{N} \\
Y_{N} \\
Z_{N}
\end{array}\right], A=\left[\begin{array}{cccccccccccc}
1 & 1 & 1 & 0 & 0 & 0 & 0 & 0 & 0 & 1 & 0 & 0 \\
0 & 0 & 0 & 1 & 1 & 1 & 0 & 0 & 0 & 0 & 1 & 0 \\
0 & 0 & 0 & 0 & 0 & 0 & 1 & 1 & 1 & 0 & 0 & 1 \\
1 & 1 & 1 & 0 & 0 & 0 & 0 & 0 & 0 & 1 & 0 & 0 \\
0 & 0 & 0 & 1 & 1 & 1 & 0 & 0 & 0 & 0 & 1 & 0 \\
\vdots & & & & & \cdots & & & & & & \vdots \\
1 & 1 & 1 & 0 & 0 & 0 & 0 & 0 & 0 & 1 & 0 & 0 \\
0 & 0 & 0 & 1 & 1 & 1 & 0 & 0 & 0 & 0 & 1 & 0 \\
0 & 0 & 0 & 0 & 0 & 0 & 1 & 1 & 1 & 0 & 0 & 1
\end{array}\right], X=\left[\begin{array}{c}
m_{11} \\
m_{12} \\
m_{13} \\
m_{21} \\
m_{22} \\
m_{23} \\
m_{31} \\
m_{32} \\
m_{33} \\
T_{x} \\
T_{y} \\
T_{z}
\end{array}\right]
$$

$N$ is number of common points. With the same manner, equation (6) can be formed to the matrix form (7).

The calculation of $X$ is based on the Least Square Adjustment that is minimizing of the sum of the weighted squares of the residuals.

$$
S \equiv \sum_{i=1}^{N}(\text { residuals })^{2}=(B-A X)^{2}
$$

To compute the value of $X$, for which $S$ becomes a minimum, one requires

$$
\frac{d S}{d X}=0
$$

The standard deviation of the observations $\sigma_{s}$ is computed as

$$
\sigma_{s}=\sqrt{\frac{1}{N-1}(B-A \bar{X})^{2}}
$$

and the standard deviation of the mean

$$
\sigma_{m}=\frac{\sigma_{s}}{\sqrt{N}}
$$




\section{$4 \quad$ Sample}

In this study, four patients were observed with CTscanner, Laser scanner, and CR photogrammetry in HUSM. The specifications of data are:

1. The GE CT sequence images the whole head with slices taken at $1.25 \mathrm{~mm}$, containing in total 204 slices (12 bits) in DICOM format.

2. The Laser scan data contains about 300.000 point clouds.

3. Six true color 24 bit images of the patient with $1600 \times 1200$ pixels with 300 DPI.

The following software tools are utilized:

1. 3D Slicer from MIT Artificial Intelligence Lab for 3D segmentation, reconstruction, and visualization of the CT data;

2. RapidForm from Inus Technology Inc. for post-processing of laser scanned data;

3. Digital Video Plotter (DVP) from DVPGS Canada for Photogrammetry process and soft-tissue's landmark identification.

When necessary we wrote our own software in Object Pascal, like program for surface-based registration, landmark-based registration and osteometric scaling.

\section{$5 \quad$ Result}

Using the set of data from one patient, the registration process is started by setting landmarks on the skin and the skull meshes. For registration purpose, only five landmarks (tragion, exocanthion left, exocanthion right, subnasale, nasion and pronasale) on skin and three landmarks (tragion left, tragion right and orbitale left) on skull are used.

Then the three landmarks are used on both skin surfaces (CT and Laser) for the initial surface-based registrations. Landmarks around the nose are chosen because that is where the features are the most marked on the human face. In a couple of seconds the registration is automatically processed. In order to assess the accuracy of the registration, measurements are made around the facial area: most vertices lie within a distance of $1.33 \mathrm{~mm}$ from the other mesh. The visualization of the surface-based registration between data from laser scanner and CT scanner is shown in Fig. 7.

The second registration was done between the 3D model and landmarks in CR photogrammetry system using $3 \mathrm{D}$ affine transformation. Using 5 landmarks as common points, the standard error of this registration is $2 \mathrm{~mm}$. The remaining misalignment comes mainly from the difference of $3 \mathrm{D}$ shapes between the 3 
sets of data and not from the registration method itself. Finally it is now possible to visualize the skull and skin data together, Fig. 8.
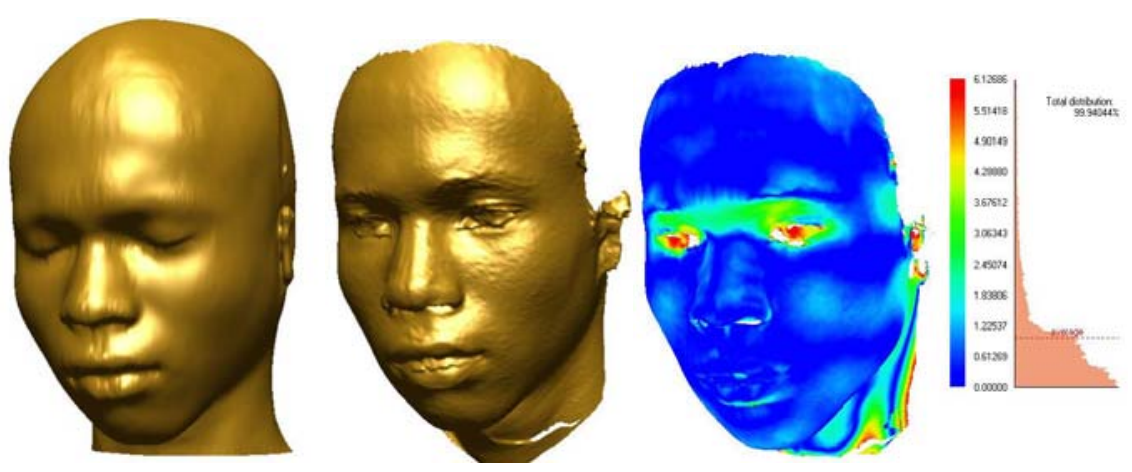

Figure 7 Skin surface from CT(a), from laser (b), differences between CT and Laser (c).
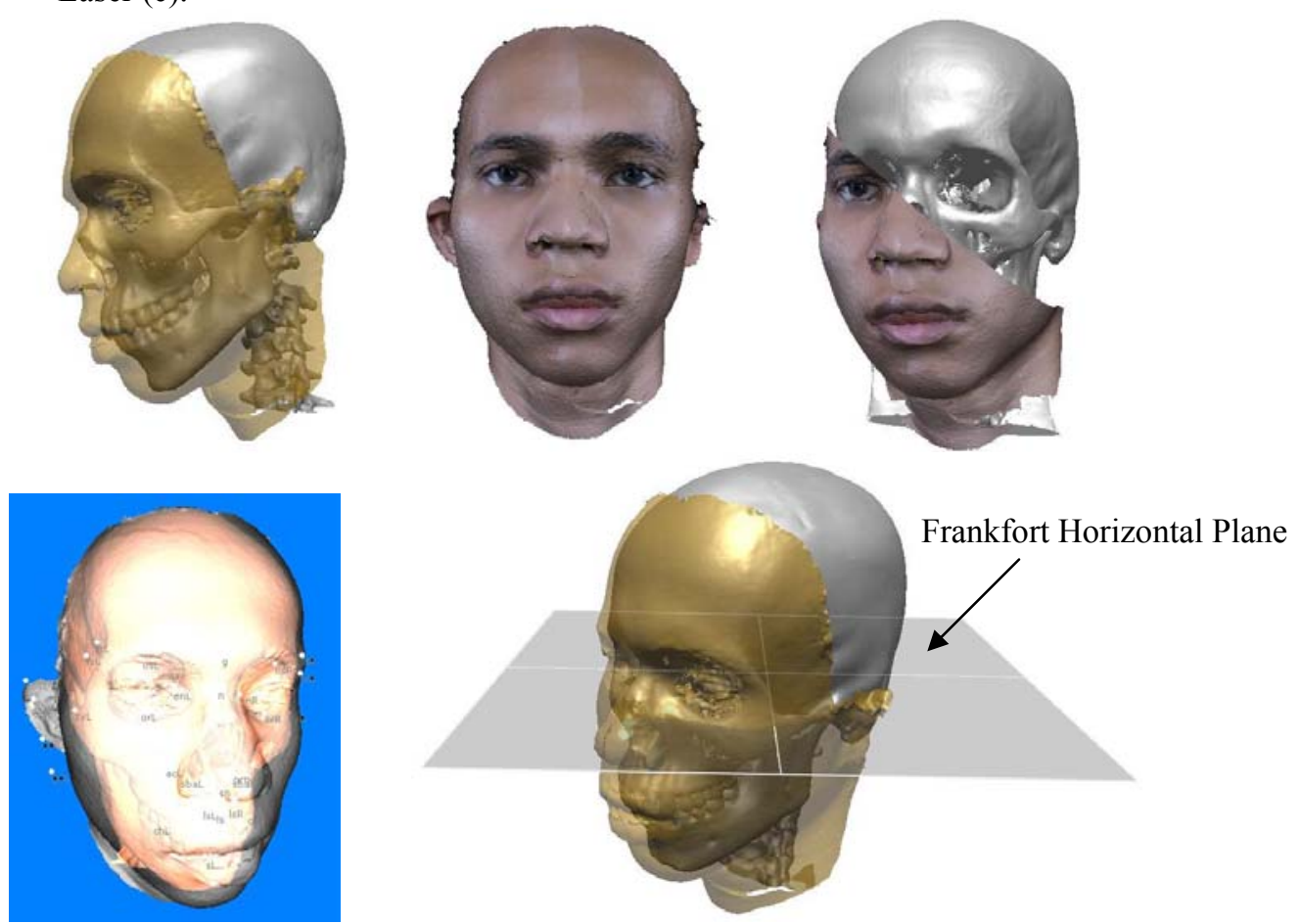

Figure 8 Integrated 3D model from CT, Laser and CR Photogrammetry.

\section{Conclusion}

In this study, two 3D surface models and one 3D landmarks obtained by three different modalities, laser scan, CT scan and CR photogrammetry have been 
attempted to superimpose. The aim of the superimposition is to produce a 3D model of a subjects' hard tissue i.e. skull, and over lay this with the subjects soft tissue and with their landmarks in one standard coordinate system. The soft tissue needs to be positioned accurately over the underlying hard tissue. The space between the two would represent the soft tissue thickness. This working goes someway to addressing these objectives. Future studies will need to address the accuracy and validity of this superimposition technique and develop a more automated approach to $3 \mathrm{D}$ objects superimposition. A registration accuracy of $1-2 \mathrm{~mm}$ at this very early stage is promising. The effect of CT scan slice thickness and the number of slices on the accuracy of superimposition needs to be calculated. This working is a step forward for storing the craniofacial spatial data in one reference system in database.

The directions of further research include: surface curvature calculation, extraction of 3-D feature lines or crest lines, and matching using 3-D deformable lines model. These steps use differential geometry of surface for automatic landmarks identification in object registration. Another function of landmarks is for generating correspondence surface points between craniofacial data for 3D statistical model building.

\section{Acknowledgement}

This research is part of a prioritized research IRPA vot. 74537 sponsored by Ministry of Science, Technology \& Innovation Environment (MOSTI) Malaysia.

\section{References}

1. Farkas, L. G. ed., Anthropometry of Head and Face. 2nd ed. New. York: Raven Press (1994).

2. Brief, J., Hassfeld, St., Münchenberg, J., Grabowski, H., Redlich, T., Walz, M., Krempien, R., Raczkowsky, J., Burgert, O., Salb, T., Rembold, U., Wörn, H., Dillmann, R. \& Mühling, J., 3-D Norm Data - the first step towards semiautomatic virtual craniofacial surgery, In: H. Lemke, M.W. Vannier, K. Inamura, A.G. Farman (eds.): Computer Assisted Radiology and Surgery. (S. 834-838.) Proceedings of the 13th International Symposium and Exhibition on Computer Assisted Radiology and Surgery, CAR'99 Paris, 23-26 June 1999 Elsevier Press, Amsterdam, Lausanne, New York, Oxford, Shannon, Singapore, Tokyo (1999).

3. Besl, P. \& McKay, N., A Method for Registration of 3-D Shapes, In IEEE. Trans. Pattern Analysis and Machine Intelligence 14, pp. 239-256 (1992). 
4. Zhang, Z., Iterative point matching for registration of freeformcurves and surfaces, In International Journal of Computer Vision 13, pp. 119-152 (1994).

5. Maintz, J. B. A. \& Viergever, M. A., A survey of medical image (1998).

6. Sakas G., Karangelis, G. \& Pommert, A., Advanced Applications of Volume Visualization Methods in Medicine, In Stergiopoulos, S., Raton, B., editors, Advanced Signal Processing Handbook: CRC Press LLC (2001).

7. Boissinat, JD., Surface reconstruction from planar crosssections, In Proceedings of IEEE Conference on Computer Vision and Pattern Recognition, pp. 393-397 (1985).

8. Lorensen, W. E. \& Cline, H. E., Marching cubes: A high resolution 3-D surface construction algorithm, In Proceedings of SIGGRAPH, pp. 163169 (1987).

9. Kolar, J. C. \& Salter, E. M. eds., Craniofacial Anthropometry: Practical Measurements of Head and Face for Clinical,Surgical, and Research. Springfield, IL: Charles C. Thomas (1997).

10. West, J., et al., Comparision and evaluation of retrospective intermodality brain image registration techniques, In Journal of Computer Assisted Tomography, 21(4), pp. 554-566 (1997).

11. Shiang T. Y., A Statistical Approach to Data Analysis and 3-D Geometric Description of the Human Head and Face, In Proceedings of the National Science Council, Republic of China, Part B: Life Sciences Vol. 23, No.1 (1999). 